\title{
Synthesis of the oxysterol, 24(S), 25-epoxycholesterol, parallels cholesterol production and may protect against cellular accumulation of newly-synthesized cholesterol Jenny Wong1, Carmel M Quinn² and Andrew J Brown*1
}

Address: ${ }^{1}$ School of Biotechnology and Biomolecular Sciences, The University of New South Wales, Sydney, Australia and ${ }^{2}$ Centre for Vascular Research at The University of New South Wales and Department of Haematology, Prince of Wales Hospital, Sydney, Australia

Email: Jenny Wong - babynekko@gmail.com; Carmel M Quinn - carmel.quinn@unsw.edu.au; Andrew J Brown* - aj.brown@unsw.edu.au

* Corresponding author

Published: 5 April 2007

Lipids in Health and Disease 2007, 6:10 doi:10.1186/1476-5IIX-6-10

This article is available from: http://www.lipidworld.com/content/6/I//0

(c) 2007 Wong et al; licensee BioMed Central Ltd.

This is an Open Access article distributed under the terms of the Creative Commons Attribution License (http://creativecommons.org/licenses/by/2.0), which permits unrestricted use, distribution, and reproduction in any medium, provided the original work is properly cited.
Received: 8 March 2007

Accepted: 5 April 2007

\begin{abstract}
Aim: The effects of 24(S),25-epoxycholesterol $(24,25 \mathrm{EC})$ on aspects of cholesterol homeostasis is welldocumented. When added to cells, 24,25EC decreases cholesterol synthesis and up-regulates cholesterol efflux genes, including ABCAI. Synthesis of $24,25 \mathrm{EC}$ occurs in a shunt of the mevalonate pathway which also produces cholesterol. Therefore, $24,25 \mathrm{EC}$ synthesis should be subject to the same negative feedback regulation as cholesterol synthesis. To date, no role has been ascribed to $24,25 \mathrm{EC}$ in light of the fact that increased accumulation of cholesterol should decrease formation of this oxysterol through feedback inhibition. This leads to the intriguing paradox: why inhibit production of an apparently important regulator of cholesterol homeostasis when it is needed most?

Methods: We used a combination of pharmacological and genetic approaches in Chinese Hamster Ovary cell-lines to investigate this paradox. Endogenous synthesis of $24,25 \mathrm{EC}$ was manipulated using partial inhibition of the enzyme, Oxidosqualene Cyclase. Changes in cholesterol and 24,25EC synthesis were determined using metabolic labelling with $\left[1-{ }^{4} \mathrm{C}\right]$-acetate, thin-layer chromatography and phosphorimaging. Transcriptional effects mediated via SREBP and LXR were analysed by luciferase reporter assays.

Results: We showed that cholesterol addition to cells lead to a rapid and preferential inhibition of $24,25 \mathrm{EC}$ synthesis. Addition of $24,25 \mathrm{EC}$ resulted in parallel inhibition of $24,25 \mathrm{EC}$ and cholesterol synthesis. Furthermore, we used a variety of approaches to examine the relationship between cholesterol and $24,25 \mathrm{EC}$ synthesis, including cell-lines with different rates of cholesterol synthesis, varying cholesterol synthetic rates by pre-treatment with a statin, or lipoprotein cholesterol loading of macrophages. In all cases, we showed that $24,25 \mathrm{EC}$ synthesis faithfully tracked cholesterol synthesis. Moreover, changes in 24,25EC synthesis exerted downstream effects, reducing SREBP transcriptional activity whilst increasing $A B C A I$ and $L X R$ transcriptional activity.

Conclusion: Our results show that 24,25EC synthesis parallels cholesterol synthesis, consistent with this oxysterol functioning as a safety valve to protect against the accumulation of newly-synthesised cholesterol (as opposed to exogenously-derived cholesterol). Considering that 24,25EC is capable of being produced in all cholesterogenic cells, we propose that production of $24,25 \mathrm{EC}$ may represent a ubiquitous defence mechanism.
\end{abstract}




\section{Background}

Oxygenated cholesterol derivatives or oxysterols are potential regulatory molecules in cholesterol homeostasis. The regulatory effect of oxysterols was first identified by Kandutsch and colleagues [1] who showed that the suppressive effect of cholesterol on its own synthesis may be mediated by endogenously-produced oxysterols. This became known as the "Oxysterol Hypothesis of Cholesterol Homeostasis" [1].

Certain oxysterols can act as suppressors of 3-hydroxy-3methylglutaryl (HMG)-CoA reductase, the rate-limiting enzyme in the mevalonate pathway which also synthesises cholesterol [2]. It has been demonstrated that oxysterols decrease the expression of cholesterol synthetic and uptake genes by suppressing sterol regulatory elementbinding protein-2 (SREBP-2) mediated transcription $[3,4]$. Moreover, certain oxysterols can act as ligands for the Liver $\times$ Receptor (LXR), which mediates transcription of cholesterol export genes, such as the ATP-binding cassette transporter, ABCA1 [5-7]. Most of the common physiological oxysterols characterised (such as the hydroxycholesterols) are derived from enzymatic oxidation of cholesterol, either from circulating lipoproteins or from synthesised cholesterol $[8,9]$.

The oxysterol $24(S), 25$-epoxycholesterol $(24,25 \mathrm{EC})$ is unique in that it is not derived from cholesterol. Instead, $24,25 \mathrm{EC}$ is produced de novo in the mevalonate pathway which simultaneously synthesizes cholesterol. 24,25EC was first characterised by Nelson, Steckbeck and Spencer [10] who showed that 24,25EC was derived from acetyl CoA via a shunt in the mevalonate pathway. Since then, exogenous addition of 24,25EC has been shown in vitro to decrease HMG-CoA reductase activity [11], to suppress SREBP processing [4], and to potently activate LXR-gene targets $[6,7,12]$. We have previously shown that statins inhibit the production of $24,25 \mathrm{EC}$ in human macrophages under basal conditions leading to decreases in LXR target gene expression including ABCA1 and ABCG1 [13]. Moreover, beneficial effects on cholesterol homeostasis have been reported in vitro [13-17] and in vivo [18-21] when endogenous synthesis of $24,25 \mathrm{EC}$ is stimulated. Two recent reviews $[22,23]$, have highlighted the interest in $24,25 \mathrm{EC}$ as a central regulator of cholesterol homeostasis [22].

Feedback regulation of cholesterol synthesis is well established [2]. When cholesterol levels in the cell are high, SREBP-processing is inhibited resulting in down-regulation of SREBP-target genes, including HMG-CoA reductase [2]. Considering that cholesterol and 24,25EC synthesis utilize the same enzymes and cofactors, synthesis of 24,25EC would be expected to decrease when cellular cholesterol accumulates and feeds back on the mevalonate pathway. This leads to the intriguing question: why inhibit production of a compound that potently regulates cholesterol homeostasis when it is needed most? To date, no publication on 24,25EC has addressed or even raised this important issue. Our current work helps to resolve this paradox by showing that although cholesterol addition potently inhibits 24,25EC synthesis, synthesis of this oxysterol tends to track cholesterol synthesis under a variety circumstances. This has lead us to propose that production of this oxysterol represents a ubiquitous defence mechanism against cellular accumulation of cholesterol that is newly-synthesised in the mevalonate pathway (as opposed to exogenously-derived cholesterol).

\section{Results \\ Cholesterol accumulation inhibits 24(S),25- epoxycholesterol synthesis}

Considering that both 24,25EC and cholesterol are synthesised in the mevalonate pathway, we would predict that 24,25EC synthesis should also be decreased upon cellular cholesterol accumulation as a consequence of feedback inhibition of the mevalonate pathway. To test this, we utilised Chinese Hamster Ovary (CHO) cells, a cellline commonly-used to study cholesterol homeostasis that has been instrumental in the identification of many of the major components of the cholesterol homeostatic machinery [24]. CHO-7 cells were cholesterol-loaded by incubation with incremental concentrations of cholesterol complexed to methyl- $\beta$-cyclodextrin in the presence of [1-14 C]-acetate. Non-saponifiable lipids were extracted, separated by thin layer chromatography and phosphorimaged. Quantification of cholesterol and 24,25EC by densitometry of the phosphorimage or by scintillation counting yielded linear responses in the standard curve for both sterols (Additional file 1). Thus, changes in cholesterol and 24,25EC synthesis were subsequently quantified by densitometry of the phosphorimage. Cholesterolloading decreased synthesis of both sterols as predicted. However, 24,25EC synthesis appeared to decrease preferentially (Fig. 1A, C). Incubation of CHO-7 cells with $24,25 \mathrm{EC}$ was more potent in inhibiting sterol synthesis than cholesterol-loading (Fig. 1Dvs. 1C) and decreased both cholesterol and 24,25EC synthesis to a similar extent (Fig. 1B, D).

The effects of added cholesterol or 24,25EC on sterol synthesis were then examined over time (Fig. 1E, F). Cholesterol-loading and 24,25EC incubation decreased the synthesis of the two sterols within hours of treatment. The preferential decrease in $24,25 \mathrm{EC}$ synthesis in response to cholesterol-loading was evident within the first two hours (Fig. 1E).

Therefore, 24,25EC synthesis is inhibited in response to added sterols. We postulate that $24,25 \mathrm{EC}$ may be func- 

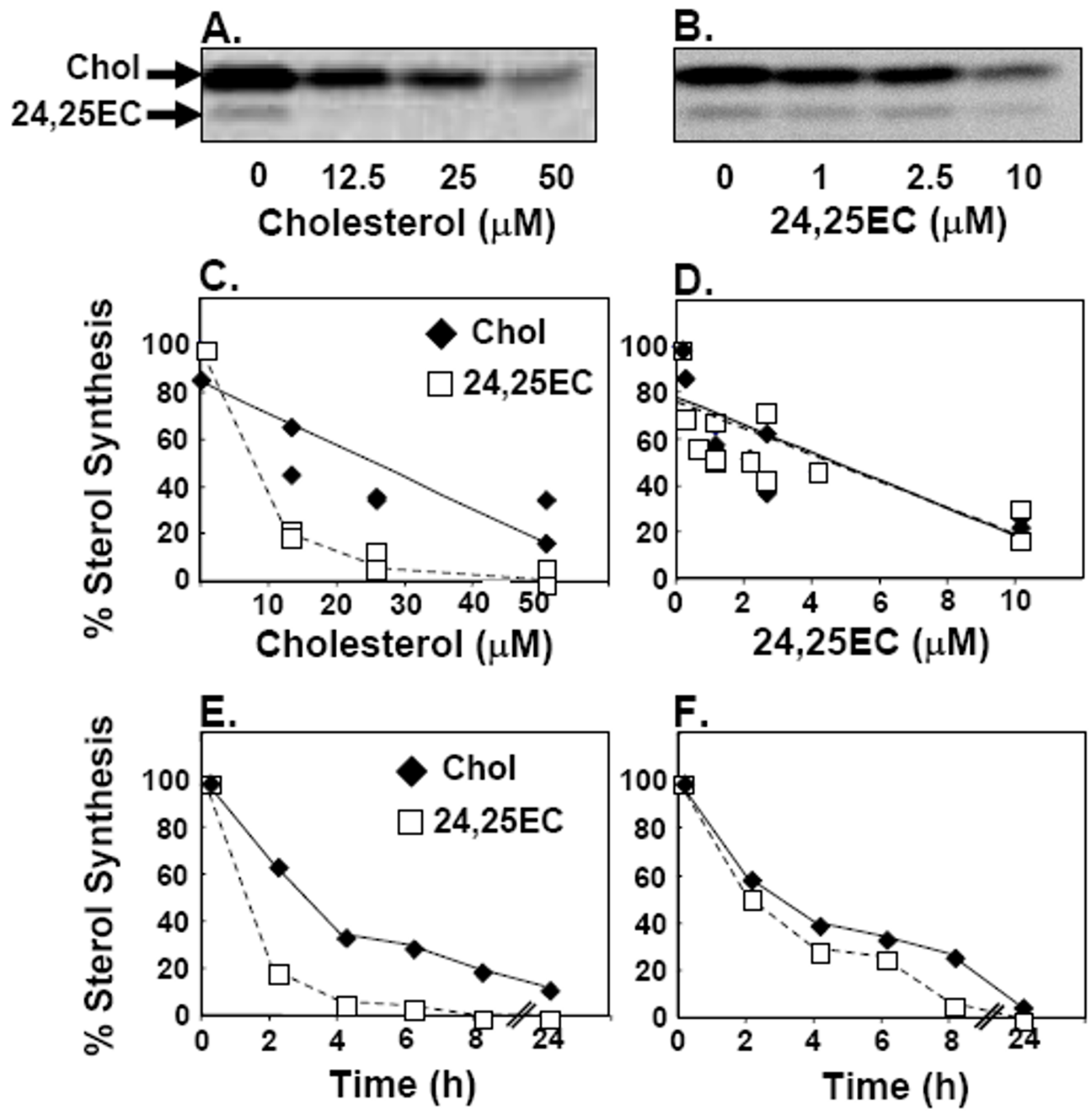

Figure I

Exogenously-added sterols feed back on cholesterol and 24,25EC synthesis. CHO-7 cells were incubated with either increasing concentrations (A) or $50 \mu \mathrm{M}(\mathbf{E})$ of cholesterol complexed to methyl- $\beta$-cyclodextrin; or either increasing concentrations (B) or $10 \mu \mathrm{M}(\mathbf{F})$ of $24,25 \mathrm{EC}$ in the presence of $\left[\mathrm{I}^{-14} \mathrm{C}\right]$-acetate for $24 \mathrm{~h}$ or $(\mathbf{E}, \mathbf{F})$ labelled $2 \mathrm{~h}$ before the indicated time of harvest. Neutral lipid extracts were separated by thin-layer chromatography and bands corresponding to authentic cholesterol and 24,25EC were visualized by phosphorimager. These phosphorimages are representative of 2 separate experiments. Bands corresponding to cholesterol and 24,25EC were quantified by densitometry. For (C, D) data sets from 2 (C) or 3 (D) separate experiments represented by Figure IA and Figure IB were pooled. Equation for the lines of best fit (C) cholesterol (solid diamond; solid line) $y=-1.35 x+84.79 ; R^{2}=0.75 ; P<0.01 ;(D) 24,25 E C$ (empty square; dashed line) $y=-5.68 x+$ 76; $R^{2}=0.58 ; P<0.0$ I; cholesterol (solid diamond; solid line) $y=-5.96 x+78 ; R^{2}=0.63 ; P<0.0 I$. For $(\mathbf{E}$, F) data sets are representativeof $\mathrm{n}=2$ separate experiments. 
tioning to protect against intracellular accumulation of cholesterol when its synthesis in the mevalonate pathway is active.

\section{Synthesis of 24(S),25-epoxycholesterol faithfully tracks cholesterol synthesis}

If 24,25EC protects against newly-synthesised cholesterol, we would predict that syntheses of these two sterols should correlate. This was observed when addition of $24,25 \mathrm{EC}$ decreased synthesis of 24,25EC and cholesterol in parallel (Fig. 1D, F). We investigated this further using a variety of Chinese Hamster Ovary cell-lines containing specific mutations in the SREBP-processing pathway which lead to altered cholesterol biosynthesis rates (details presented in [25]). Regardless of the mutation, synthesis of 24,25EC was correlated with cholesterol synthesis (Fig. 2A, B).

In another experimental approach, the rate of cholesterol synthesis was varied in CHO-7 cells by pre-incubating with increasing concentrations of the archetypal statin, compactin. Statins decrease cholesterol synthesis by inhibiting HMG-CoA reductase (Fig. 3A). The resulting cholesterol depletion induces SREBP activation [26] and consequently up-regulates expression of key rate-limiting cholesterol biosynthetic enzymes, including HMG-CoA reductase $[13,25]$ (Fig. 3B). Hence, after pre-incubation when compactin is removed, there is an increase in the rate of cholesterol synthesis in the mevalonate pathway (Fig. 3C). The induction in cholesterol synthesis increased by up to four-fold and yet again was closely tracked by 24,25EC synthesis (Fig. 3D).

In our final approach, we studied the effects of lipoprotein cholesterol loading on cholesterol and 24,25EC synthesis in macrophages, a cell-type particularly relevant to the etiology of atherosclerosis. For these experiments, we utilized the human macrophage-like cell-line, THP-1 [13]. Cholesterol loading of THP-1 human macrophages with acetylated LDL decreased both cholesterol and 24,25EC synthesis in unison (Fig. 4A, B).

Taken together, these positive correlations between cholesterol and 24,25EC synthesis and the potent suppressive effect of added 24,25EC on cholesterol synthesis (Fig. 1B, 1D) support its role as a feedback regulator when cholesterol is being actively synthesised.

\section{4(S),25-epoxycholesterol regulates SREBP- and LXR- mediated transcription}

Addition of certain oxysterols including $24,25 \mathrm{EC}$, are known to block SREBP-2 activation in the cell $[3,4]$. To assess changes in gene transcription, we employed luciferase reporter constructs containing tandem repeats of the motif at which transcriptional changes take place. The

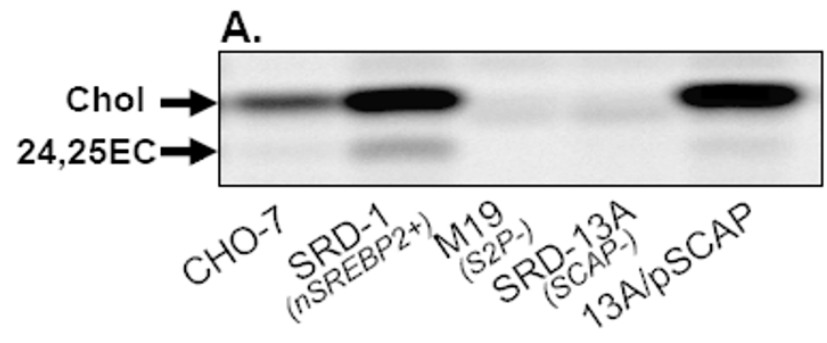

B.

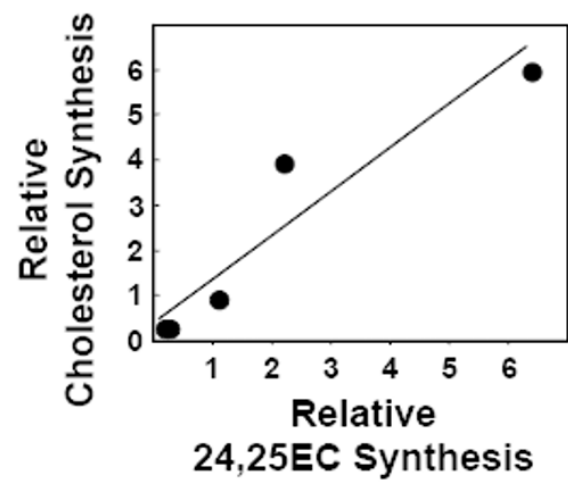

Figure 2

Endogenously-produced 24,25EC correlates with cholesterol synthesis in different $\mathrm{CHO}$ cell-lines. (A) Various Chinese Hamster Ovary cell-types with different abilities to synthesise cholesterol were incubated in the presence of [I-I4C]-acetate for $24 \mathrm{~h}$. KEY: I. CHO-7; 2. SRD-I; 3. MI9; 4. SRD-I3A; 5. I3A/PSCAP. For more details about these cell-lines, see [25]. Neutral lipid extracts were separated by thin-layer chromatography and bands corresponding to authentic cholesterol and $24,25 \mathrm{EC}$ were visualized by phosphorimager and quantified by densitometry. Equations for the lines of best fit (B) $y=0.97 x+0.39 ; R^{2}=0.89 ; P<$ 0.03 . Values are means from 3 separate experiments set relative to $\mathrm{CHO}-7$ cells which was assigned an arbitrary value of I.

effect of added 24,25EC on SREBP-mediated transcription was examined using a luciferase reporter construct containing 6 tandem copies of the classical sterol response element from the promoter of the LDL receptor, ATCACCCCAC (SRE-luc) $[27,28]$. Hence, changes in SRE-luc reporter activity reflect SREBP-mediated transcription. $24,25 \mathrm{EC}$ is a potent activator of LXR-target genes $[6,12]$ including the cholesterol efflux gene, ABCA1 $[29,30]$. To assess LXR-mediated gene transcription, we used a luciferase reporter construct containing a $1 \mathrm{~kb}$ fragment of the human ABCA1 promoter [30] as well as a luciferase reporter containing 3 repeats of the minimal DR4 motif (LXRE-luc). In agreement with our previous findings [25], addition of increasing concentrations of 24,25EC lead to a decrease in SRE reporter activity and to reciprocal 
A. Cholesterol Synthesis

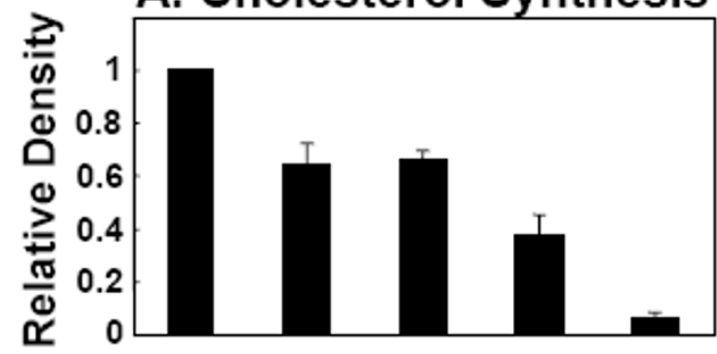

\section{B. HMG-CoA reductase}

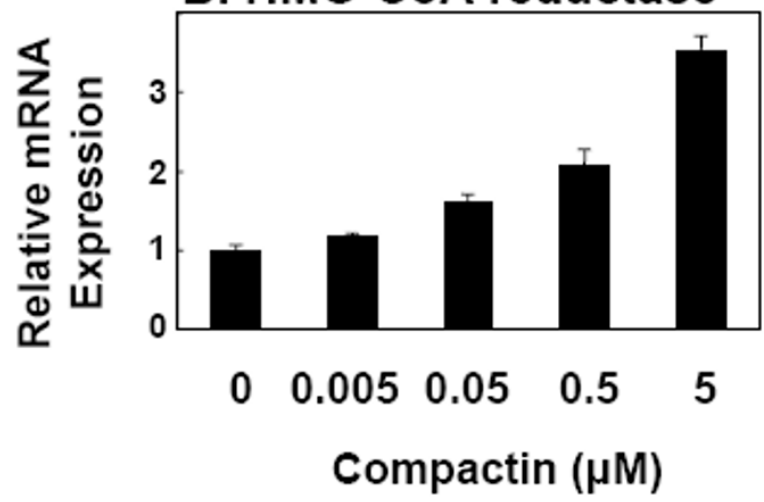

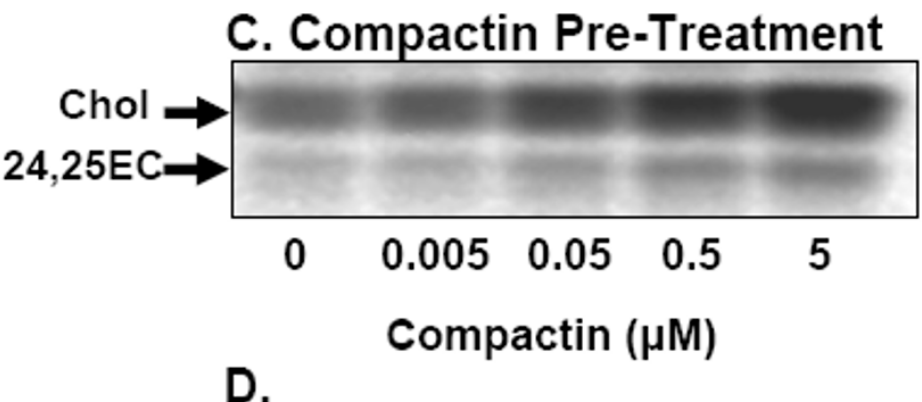

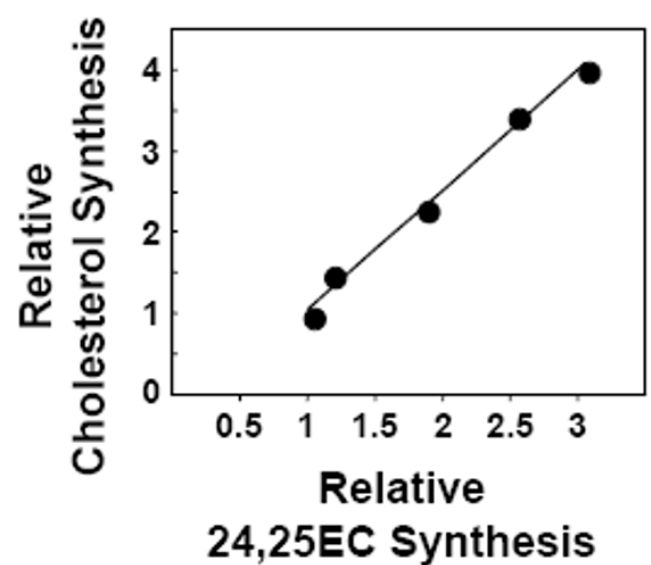

Figure 3

Endogenously-produced 24,25EC correlates with cholesterol synthesis when cholesterol synthesis is stimulated by statin-pre-treatment. (A) $\mathrm{CHO}-7$ cells were incubated with the indicated concentrations of compactin in the presence of [ $1-14 \mathrm{C}]$-acetate for $24 \mathrm{~h}$. Neutral lipid extracts were separated by thin-layer chromatography and bands corresponding to authentic cholesterol and 24,25EC were visualized by phosphorimager and quantified by densitometry. Values are means+SEM from 3 separate experiments. $(\mathbf{B}, \mathbf{C})$ CHO-7 cells were pre-incubated with the indicated concentrations of compactin for 24 h. (B) mRNA levels for HMG-CoA reductase was measured using QRT-PCR. Data are presented relative to vehicle-treated controls and are means+SEM (3 replicate cultures representative of 2 separate experiments). (C) Cells were then incubated in the presence of $\left[\mathrm{I}-{ }^{14} \mathrm{C}\right]$-acetate for $2 \mathrm{~h}$. Neutral lipid extracts were separated by thin-layer chromatography and bands corresponding to authentic cholesterol and 24,25EC were visualized by phosphorimager and quantified by densitometry. Equations for the lines of best fit $(D) y=1.47 x-0.42 ; R^{2}=0.99 ; P<0.001$. Values are means from 2 separate experiments set relative to the vehicle-treated control condition which was assigned an arbitrary value of $\mathrm{I}$.

increases in ABCA1 and LXRE-luc reporter activity (Fig. $5 \mathrm{~A}-\mathrm{C})$ ).

It is well documented that addition of $24,25 \mathrm{EC}$ has marked effects on parameters associated with cholesterol homeostasis $[6,13,14,25]$. We aimed to determine if endogenous 24,25EC also has the expected effects on SREBP- and LXR- mediated transcription. A specific inhibitor of the enzyme 2,3-oxidosqualene cyclase (OSC), GW534511X, was used to partially inhibit the activity of this enzyme, hence increasing the amount of 24,25EC produced in the shunt pathway (illustrated in Fig. 6A, B). We and others have shown that use of high OSC inhibitor concentrations increase production of 2,3(S):22(S),23dioxidosqualene (DOS) and almost completely abolish cholesterol synthesis $[13,14,17]$. Since these may produce confounding effects, lower inhibitor concentrations were used. In agreement with previous studies [13-16,18,20], we found that partial OSC inhibition increased 24,25EC synthesis and decreased cholesterol synthesis (Fig. 6C).

When endogenous 24,25EC synthesis was increased by partial OSC inhibition (Fig. 6C), SREBP-reporter activity was decreased (Fig. 6D). In addition, we assessed SREBPprocessing through use of an SREBP-reporter fusion construct [26] which confirmed that endogenously-produced 24,25EC decreased SREBP-processing (data not shown).

ABCA1 luciferase reporter activity increased in accordance with the increase in 24,25EC synthesis when OSC was partially inhibited by $0.1 \mathrm{nM}$ and $1 \mathrm{nM}$ GW534511X (Fig. $6 \mathrm{E})$. To confirm that ABCA1 promoter activity was indeed 


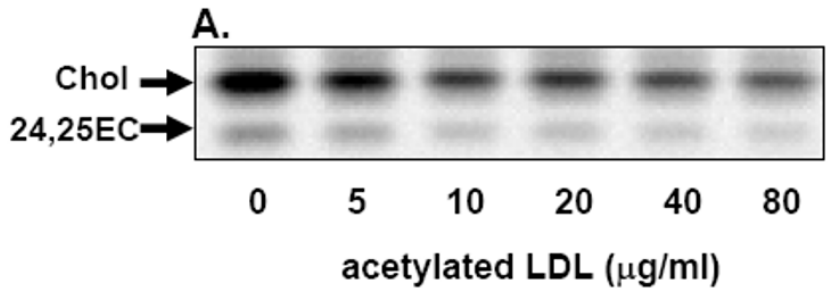

B.

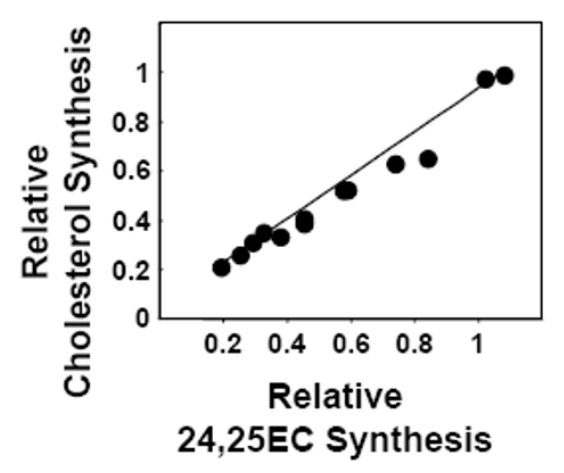

Figure 4

Endogenously-produced 24,25EC correlates with cholesterol synthesis when macrophages are cholesterol-loaded. (A) THP-I monocytes differentiated into macrophages for $3 \mathrm{~d}$ in $50 \mathrm{ng} / \mu \mathrm{l}$ of PMA. THP-I macrophages were loaded with acetylated LDL for $24 \mathrm{~h}$ whilst labelling with $\left[1-{ }^{14} C\right]$-acetate. Neutral lipid extracts were separated by thin-layer chromatography and bands corresponding to authentic cholesterol and 24,25EC were visualized by phosphorimager and quantified by densitometry. Neutral lipid extracts were separated by thin-layer chromatography and bands corresponding to authentic cholesterol and 24,25EC were visualized by phosphorimager and quantified by densitometry. Equations for the lines of best fit (B) $y=0.88 x ; R^{2}=$ $0.97 ; P<0.0001$. Values are pooled from 2 separate experiments set relative to the vehicle-treated control condition which was assigned an arbitrary value of $\mathrm{I}$.

the result of increased endogenous synthesis of LXR ligand 24,25EC, two approaches were employed. Firstly, the wild-type ABCA1 reporter construct was mutated at the LXR response element, DR4 motif (DR4 mutant). Reporter activity of the DR4 mutant construct was lower than that of the wild-type construct and displayed no response to changes in endogenous 24,25EC synthesis (Fig. 6E). Secondly, we observed similar effects of GW534511X treatment on LXRE-luc activity as compared with ABCA1 reporter activity (Fig. 6F). This confirms that the changes induced by GW534511X were mediated through the LXR response element and are most likely due to endogenous $24,25 \mathrm{EC}$ synthesis. This result also suggests that the effect of endogenously-produced 24,25EC should be applicable to other LXR-target genes.

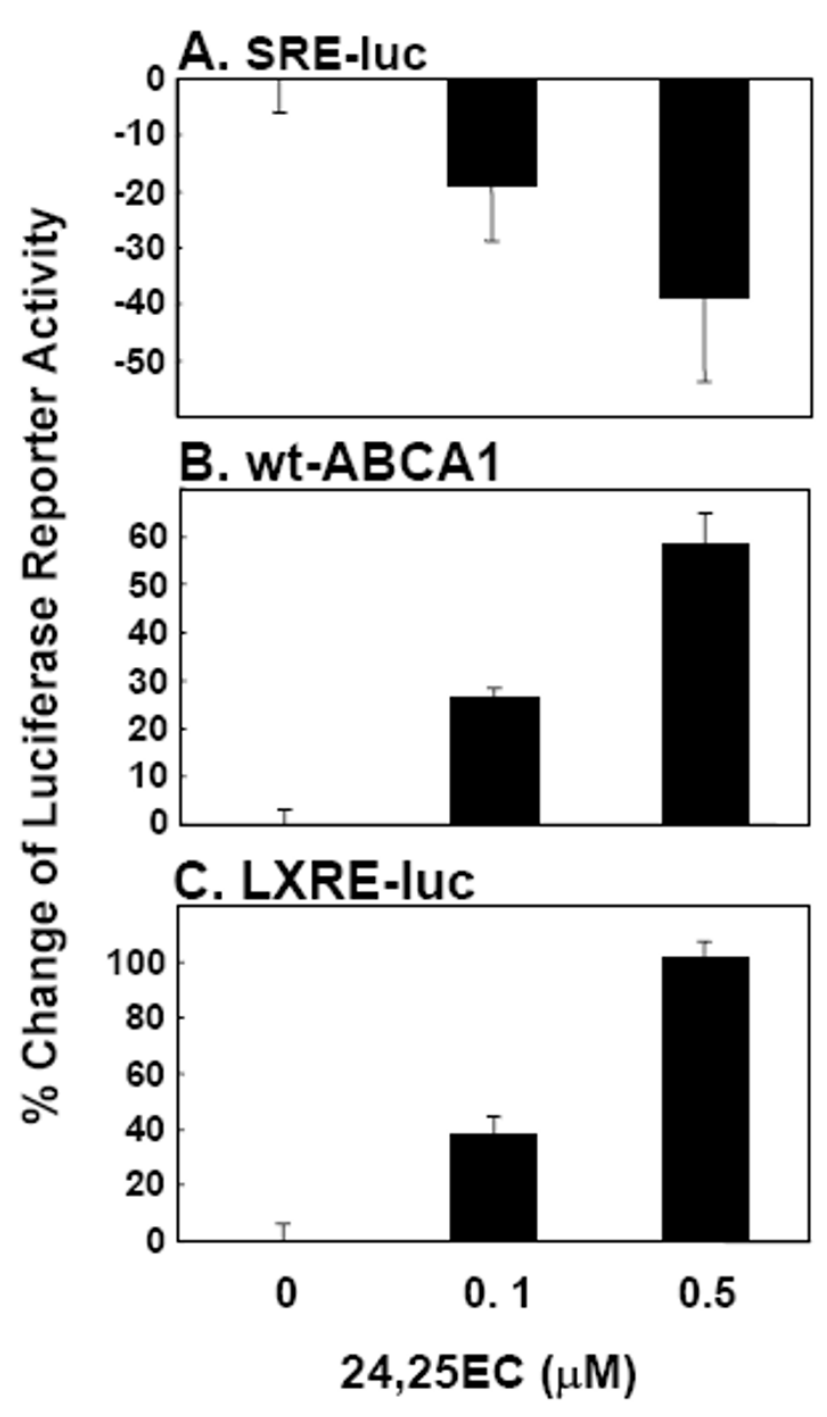

Figure 5

Added 24(S),25-epoxycholesterol regulates SREBPand LXR-mediated transcription. (A-C) $\mathrm{CHO}-7$ cells were transiently transfected for $24 \mathrm{~h}$ with phRL-TK Renilla internal control plasmid together with either (A) SRE-luc, (B) PGL3-hABCAI wild-type, (C) LXRE-luc. Following transfection, cells were incubated for $24 \mathrm{~h}$ in the absence or presence of indicated concentrations of 24,25EC. Data are presented as percentage change relative to the vehicletreated control condition. (A,B) Values are mean+SEM $(n=$ 3 separate experiments). (C) Values aremean+SEM ( $n=3$ replicate cultures representative of 3 separate experiments).

Collectively, our results support the idea that endogenously-produced 24,25EC may act as a feedback regulator to protect the cell against the cholesterol accumulation of newly-synthesized cholesterol. 

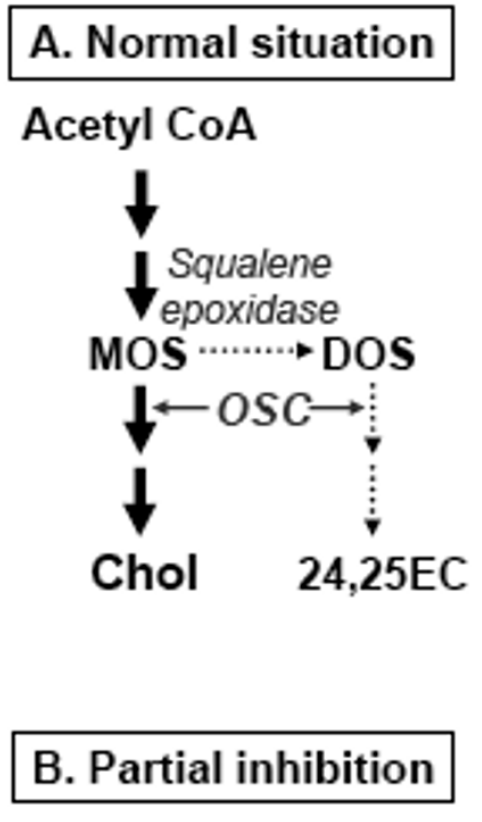

Acetyl CoA

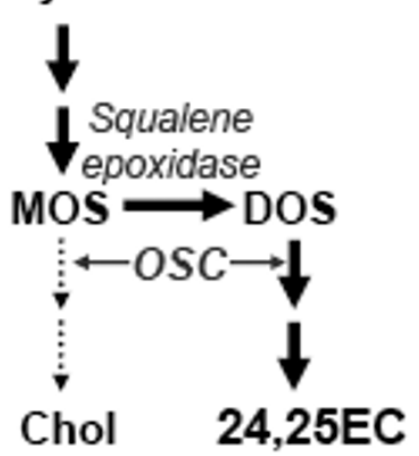

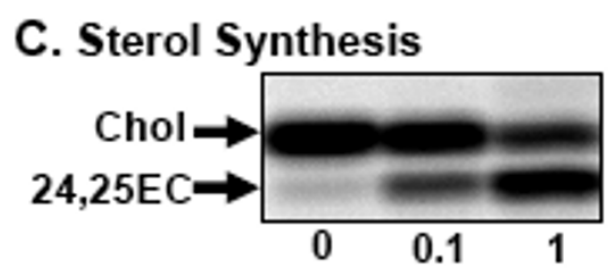

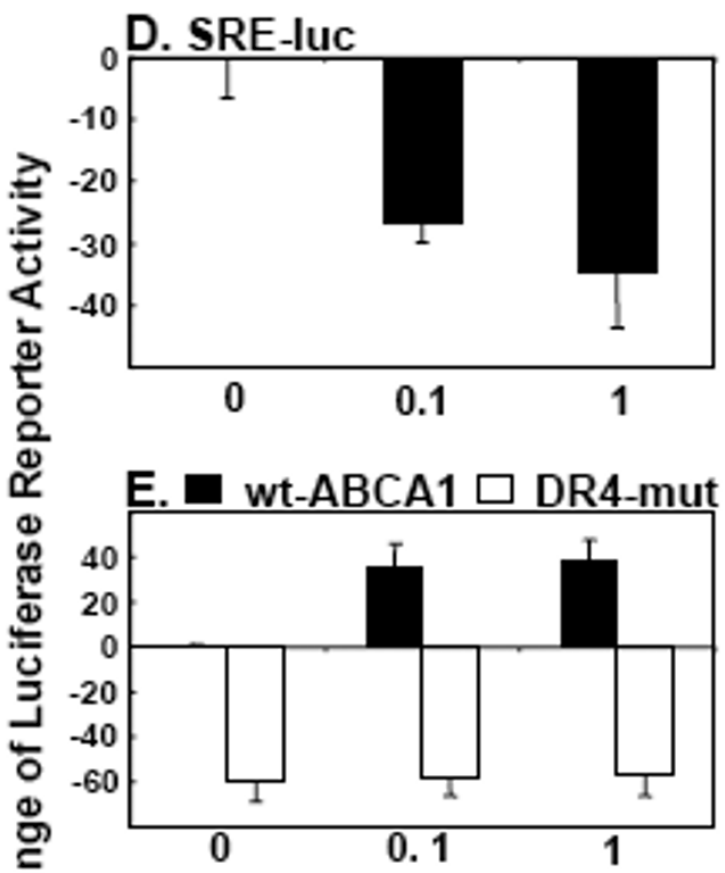

F. LXRE-luc

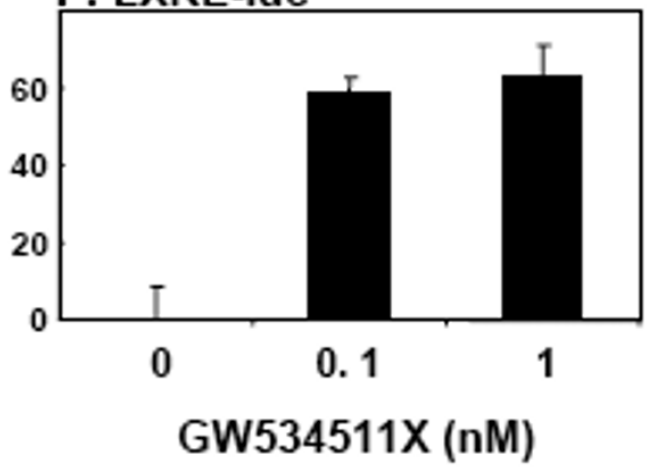

Figure 6

Endogenous 24(S),25-epoxycholesterol regulates SREBP- and LXR-mediated transcription. (A) Under normal conditions, cholesterol synthesis is favoured but some $24,25 \mathrm{EC}$ is still produced. (B) Since oxidosqualene cyclase (OSC) preferentially cyclises 2,3(S):22(S),23-dioxidosqualene (DOS) over 2,3(S)-monooxidosqualene (MOS), partial inhibition of OSC favours synthesis of 24,25EC. (C) CHO-7 cells were incubated with increasing concentrations of GW5345 I IX in the presence of $\left[\mathrm{I}-{ }^{14} \mathrm{C}\right]$-acetate for $24 \mathrm{~h}$. Neutral lipid extracts were separated by thin-layer chromatography and bands corresponding to authentic cholesterol and 24,25EC were visualized by phosphorimager. This phosphorimage is representative of 3 separate experiments. (D-F) CHO-7 cells were transiently transfected for $24 \mathrm{~h}$ with phRL-TK Renilla internal control plasmid together with either (D) SRE-luc, (E) PGL3-hABCAI wild-type or DR4 mutant, (F) LXRE-luc. Following transfection, cells were incubated for $24 \mathrm{~h}$ in the absence or presence of indicated concentrations of GW5345I IX. Data are presented as percentage change relative to (E) the PGL3-hABCAI wild-type construct vehicle-treated control condition or (D, F) vehicle-treated control condition. (D, E, F) are means+SEM( $n=3$ separate experiments); for $(E)$ DR4 mutant are means+halfrange $(n=2$ separate experiments). 


\section{Discussion}

$24,25 \mathrm{EC}$, produced de novo in a shunt of the mevalonate pathway, is a promising physiological oxysterol mediator of cholesterol homeostasis $[22,23]$. The shunt pathway runs parallel to cholesterol synthesis. Thus enzymes for the synthesis of both cholesterol and 24,25EC are shared and subject to the same regulation. Paradoxically, synthesis of 24,25EC decreases when cholesterol accumulates and feeds back, preventing activity of the mevalonate pathway i.e. when cellular cholesterol is high and the need for 24,25EC should be greatest. Our current work helps to clarify this paradox by proposing a previously unrecognized role of $24,25 \mathrm{EC}$ as a defence mechanism against cellular accumulation of newly-synthesized cholesterol as opposed to exogenously-derived cholesterol from lipoproteins. Below we present several lines of evidence in support of this contention.

Firstly, we showed that addition of pure cholesterol to cells preferentially shut down synthesis of 24,25EC (Fig. $1 \mathrm{C}, \mathrm{E})$. Moreover, lipoprotein-cholesterol loading of macrophages also decreased 24,25EC synthesis (Fig. 4A). These results confirm our prediction that 24,25EC's role can not be to protect against accumulation of exogenous cholesterol and suggests a feed-back system designed to preferentially attenuate levels of this oxysterol when cholesterol synthesis is not required.

We further explored the role of $24,25 \mathrm{EC}$ synthesis as a feed-back mechanism by employing four different experimental approaches (Fig. 1B, Fig. 2B, 3D, 4B). We found that synthesis of 24,25EC faithfully tracks cholesterol synthesis in each case. Firstly, we suppressed the activity of the mevalonate pathway by addition of exogenous 24,25EC. Both cholesterol and 24,25EC synthesis decreased in unison and the effects observed were dependent on concentration and time (Fig. 1D, F). Secondly, through the use of mutant $\mathrm{CHO}$ cell-lines which exhibit varying rates of cholesterol synthesis, we found that 24,25EC synthesis follows cholesterol synthesis (Fig. 2B). Thirdly, when the biosynthetic activity of the mevalonate pathway was increased by statin-pre-treatment, we also observed parallel increases in cholesterol and 24,25EC synthesis (Fig. 3D). Finally, cholesterol-loading of the human macrophage cell-line, THP-1, produced parallel decreases in cholesterol and 24,25EC synthesis (Fig. 4B).

We also showed that synthesised 24,25EC had the expected downstream effects on LXR- and SREBP-mediated gene transcription. Induction of endogenous 24,25EC synthesis with the OSC inhibitor GW534511X activated ABCA1 transcription (Fig. 6E) in agreement with previous studies which have reported increased ABCA1 mRNA levels in response to partial inhibition of OSC
$[13,14,17]$. The reciprocal effects were observed for SREBP-mediated gene transcription (Fig. 6D) which decreased as $24,25 \mathrm{EC}$ synthesis increased. Again, this is in keeping with previous studies $[4,14]$. The magnitude of effects observed in these luciferase reporter assays is similar to those observed in response to sub-micromolar concentrations of added 24,25EC (Fig. 5).

Together, the parallel relationships observed between $24,25 \mathrm{EC}$ and cholesterol synthesis as well as the downsteam effects of increased 24,25EC synthesis can be rationalized if $24,25 \mathrm{EC}$ represents 'a safety-valve' for protecting against the accumulation of newly-synthesized cholesterol (Fig. 7). Where precursors of cholesterol synthesis diverge to form 24,25EC, this oxysterol can then be utilised as a regulatory molecule to decrease cholesterol synthesis, inhibit SREBP-2 mediated transcription, and to upregulate cholesterol efflux processes.

As both 24,25EC and cholesterol are synthesised in the mevalonate pathway, any cell that can synthesize cholesterol also has the capacity to generate $24,25 \mathrm{EC}$. So far, several cell-types have been shown to generate 24,25EC (including hepatocytes [15,17] (Additional file 2), macrophages $[13,14]$ (Fig. 4A), fibroblasts and lung cells [31]). Spencer et al. [32] previously estimated that the human liver contains $10-30 \mu \mathrm{M}$ of $24,25 \mathrm{EC}$. At this concentration range the activity HMG-CoA reductase could be suppressed by $50 \%$ in fibroblast cell culture [32].

The evidence presented herein is, of necessity, correlational. The use of genetic manipulation to test the idea that 24,25EC may represent a ubiquitous defence mechanism against newly-synthesized cholesterol is problematic, since synthesis of cholesterol and 24,25EC occur concurrently using the same enzymes. However, some in vivo evidence for this hypothesis was provided by Zhang et al. [19], who found that 24,25EC was relatively enriched in the nuclear fraction of rat liver homogenates, placing it in the correct location to act as an in vivo LXR ligand. Furthermore, $1 \mathrm{~h}$ after administering a bolus of mevalonic acid to rats, hepatic levels of 24,25EC doubled, in contrast to the other oxysterols measured. These observations are consistent with 24,25EC being produced in response to an overactive mevalonate pathway and supports the hypothesis that it can protect the cell from accumulation of newly-synthesized cholesterol.

Previously, we speculated that human macrophages may produce ligands for LXR, depending on the supply of exogenous cholesterol versus the activity of the shunt pathway [13]. When cholesterol status is sufficient, feedback inhibition of the mevalonate pathway occurs and enzymatic conversion of cholesterol to oxysterols may be the dominant mechanism for up-regulating cholesterol 


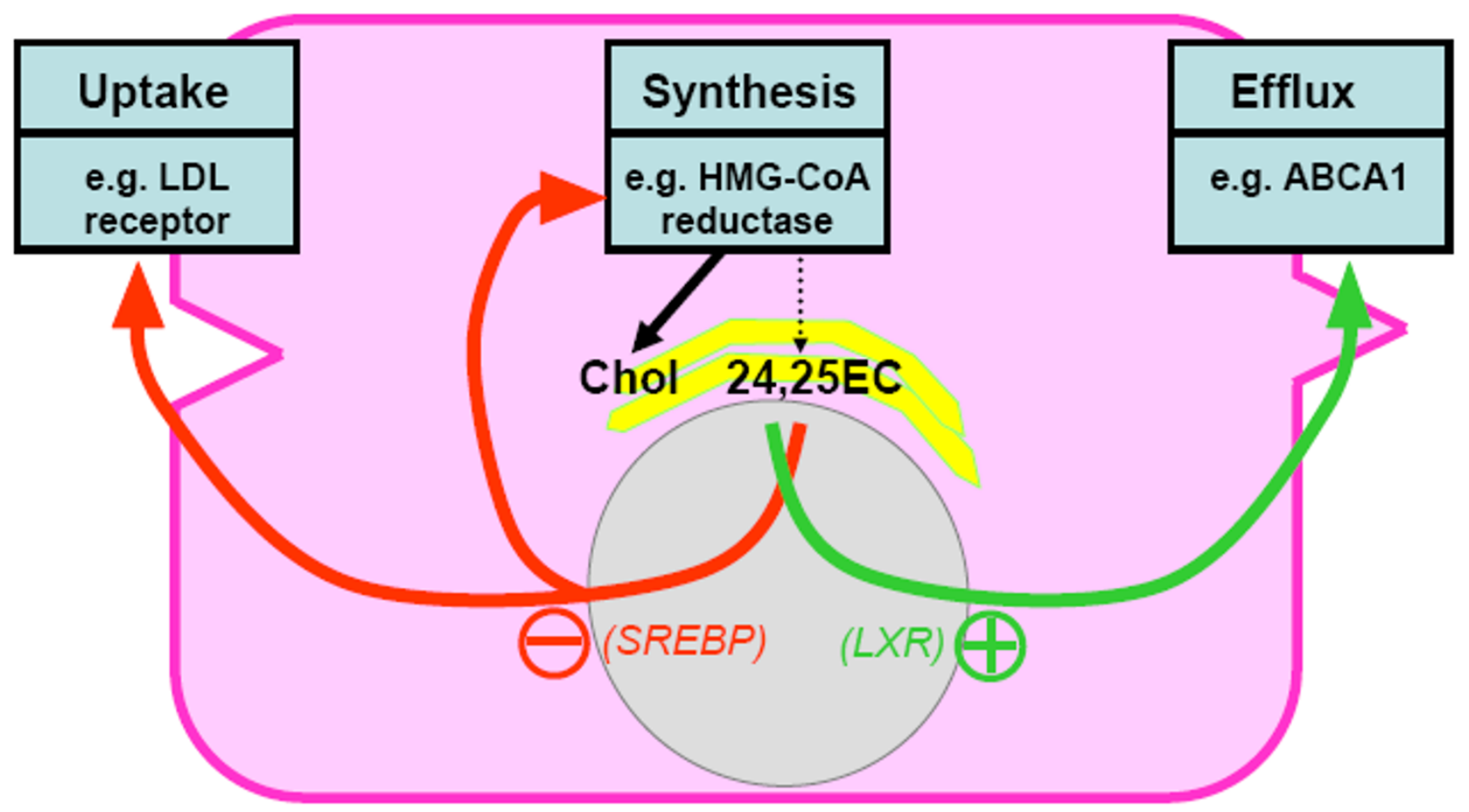

Figure 7

A working hypothesis showing 24,25EC's role in protecting against accumulation of newly-synthesised cholesterol. Synthesis of cholesterol is accompanied by synthesis of $24,25 \mathrm{EC}$ in the same pathway. $24,25 \mathrm{EC}$ can inhibit cholesterol uptake and synthesis by suppressing SREBP activation. 24,25EC can also promote cholesterol efflux by serving as a ligand for $L X R$ target genes, including $A B C A I$.

efflux genes like ABCA1, overriding any effects of 24,25EC. Indeed, when THP-1 macrophages were loaded with acetylated LDL, we observed corresponding decreases in cholesterol and 24,25EC synthesis (Fig. 4A). However, the conversion of cholesterol to oxysterols will be cell-type specific, for example, another LXR ligand 24(S)-hydroxycholesterol, is synthesized exclusively in the brain and thus probably does not function as a regulatory molecule in other cell-types [8]. In cells capable of synthesizing oxysterols from cholesterol, we propose that $24,25 \mathrm{EC}$ may serve as an auxiliary mechanism for when cholesterol synthesis is high. In cell-types unable to convert cholesterol into oxysterol ligands, we propose that $24,25 \mathrm{EC}$ may play a greater role in regulating cholesterol homeostasis.

In summary, this is the first study to rationalize the production of 24,25EC as a ubiquitous defence mechanism against newly-synthesized cholesterol. Given the role of $24,25 \mathrm{EC}$ in cholesterol homeostasis and the importance of OSC in determining its endogenous production, it is not surprising that this enzyme is of considerable interest as a pharmacological target $[14,17,22,23]$. Funk and
Landes [33] reported that treatment of hamsters and dogs with OSC inhibitors produced various histopathological lesions, including early-stage cataracts. However, these authors concluded that all adverse effects described were exaggerated at the high dosage levels employed and suggested that use of lower doses of hepatosensitive OSC inhibitors should reduce the probability of adverse sideeffects in humans [33]. Instead of blocking the entire mevalonate pathway with statins, the idea of manipulating a pre-existing and self-regulating pathway for the production of a physiological oxysterol mediator, that can augment cholesterol removal and decrease uptake and synthesis, is attractive and warrants further evaluation.

\section{Methods \\ Materials}

Chemicals and reagents used are listed below with the supplier. From GE Healthcare (formerly Amersham Biosciences): [1-14C]-acetic acid, sodium salt (specific activity: $56 \mathrm{mCi} / \mathrm{mmol}$ ). From Invitrogen: Dulbecco's modified Eagle's medium/Ham's F-12 medium (50/50 mixture); RPMI 1640; L-glutamine; Lipofectamine 2000; new born calf serum; fetal calf serum; penicillin/strepto- 
mycin. From Promega: phRL-TK Renilla plasmid. From Sigma: compactin (also called mevastatin); phorbol 12myristate 13-acetate (PMA); TriReagent; oligonucleotides were synthesized by Sigma-Genosys. From Steraloids: cholesterol; 24(S),25-epoxycholesterol. Other reagents were: analytical- or HPLC-grade solvents (EM Science); UltimaGold scintillation fluid (Packard Bioscience). Lipoprotein-deficient serum (LPDS) was prepared from new born calf serum or human serum [34]. Acetylation of low density lipoprotein (LDL) to acetylated LDL was prepared as previously described [35]. Chinese Hamster Ovary celllines, were generously provided by Drs. Michael S. Brown and Joseph L. Goldstein (UT Southwestern, Dallas). A reporter construct (pGL3-hABCA1) containing a fragment of the human ABCA1 promoter ( $-928 \mathrm{bp}$ to $+101 \mathrm{bp})$ was a kind gift from Dr Alan Tall (Columbia University, New York). Another reporter construct (pGL3-TK) was donated by Dr Malcolm Lyons (Western Australian Institute of Medical Research). The 2,3-oxidosqualene cyclase (OSC) inhibitor, GW534511X, was kindly provided by Glaxo Smith-Kline.

\section{Cell Culture}

All cell types used were grown at $37^{\circ} \mathrm{C}$ in a $5 \% \mathrm{CO}_{2}$ atmosphere. CHO-7 cells were cultured in Dulbecco's modified Eagle's medium/Ham's F-12 medium (1:1 mixture). These and other Chinese Hamster Ovary cell-lines were maintained as described [25]. THP-1 human monocytes were cultured in RPMI 1640 plus 10\% (v/v) FCS supplemented with penicillin/streptomycin $100 \mathrm{U} / 100 \mu \mathrm{g} /$ $\mathrm{ml}$ and L-glutamine ( $2 \mathrm{mM})$. Differentiation into macrophages was achieved by addition of PMA (50 ng/ $\mu \mathrm{l}$ ) for three days. Treatments were conducted for $24 \mathrm{~h}$ in media containing 5\% (v/v) LPDS. All treatments were added to cells either in absolute ethanol, dimethyl sulfoxide or water and compared with vehicle-only controls. No celltoxicity was observed for any treatment at the concentrations employed.

Cholesterol and 24(S),25-Epoxycholesterol synthesis assay Cells were metabolically labelled with [1-14C]-acetic acid for 2 or $24 \mathrm{~h}$ during treatments as previously described [13]. THP-1 macrophages were loaded with acetylated LDL whilst labelling with $\left[1-{ }^{14} \mathrm{C}\right]$-acetic acid for $24 \mathrm{~h}$. Cells were harvested and cell protein was determined by the bicinchoninic acid assay (Pierce). Samples were saponified and standardised for equal protein loading ( $150 \mu \mathrm{g}$ protein) and neutral lipid extracts were separated by thin-layer chromatography. Bands corresponding to authentic cholesterol and 24,25EC were visualized using the FLA-5100 phosphoimager (Fujifilm, Tokyo, Japan) (18-72 h exposure). Quantification of bands by densitometry was conducted with Sciencelab ImageGauge 4.0 Software (Fujifilm). Positive identification of the band corresponding to $24,25 \mathrm{EC}$ has previously been performed chemically and by mass spectrometry (see supplemental data of reference [13]). To validate our results based on densitometry of the phosphorimage, we also performed scintillation counting for an experiment that established linearity of responses (Additional file 1). We show that increasing levels of $\left[{ }^{14} \mathrm{C}\right]$-cholesterol and $\left[{ }^{14} \mathrm{C}\right]-24,25 \mathrm{EC}$ give proportional increases in response using both methods of quantification. Following thin layer chromatography, bands corresponding to authentic cholesterol and 24,25EC were cut and eluted with methanol:peroxide free diethyl ether $(1: 1) \mathrm{v} / \mathrm{v}$. Radioactivity was determined by scintillation counting (Packard Tri-Carb 2100 TR Liquid Scintillation Analyzer) using UltimaGold scintillation fluid (5 ml).

\section{Plasmid constructs}

Mutations in the LXR response element (DR4 motif) in a fragment of the hABCA1 promoter that is linked to the firefly luciferase reporter gene [30] was generated using the Quickchange site-directed mutagenesis kit (Stratagene) [25] and sequences were verified. The $6 \times$ SRE-luciferase reporter has been previously described [25]. A $3 \times$ LXRE-luciferase reporter (LXRE-luc) was constructed using a similar approach [25]. Briefly, the $3 \times$ LXRE-luc insert was constructed by annealing complementary sequences of a $3 \times$ repeat containing the DR4 motif (underlined) "tgaatgaccagcagtaacctcagc" [36]; and KpnI and XhoI restriction sites synthesized at the 5' and 3 ' ends respectively (Sigma-Genosys). The annealed insert and a pGL3-TK vector were then sequentially digested with their respective restriction enzymes and both digested insert and vector were then ligated with T4 DNA ligase and transformed into the DH5 $\alpha$ E. coli strain. Positive clones were verified by sequencing.

\section{Human ABCAI promoter activity assay}

Promoter analysis was performed as previously described [25]. Briefly, reporter plasmids (250 ng/well) were transfected for $24 \mathrm{~h}$ into cells using Lipofectamine $2000(1 \mu \mathrm{l} /$ well). The phRL-TK Renilla internal control plasmid (25 $\mathrm{ng} /$ well) was co-transfected for normalization of transfection efficiency. After treatment $(24 \mathrm{~h})$, cells were washed and resuspended in $100 \mu \mathrm{l}$ of $1 \times$ passive lysis buffer (Promega). Luciferase assays were performed using the Dual Luciferase Assay Reporter System according to the manufacturer's instructions in a Veritas luminometer (Turner Designs). Results were expressed as changes in luciferase activity relative to vehicle-treated controls or the wild-type ABCA1 vehicle-treated control condition.

\section{Reverse Transcriptase PCR and Quantitative Real-time PCR}

Cells were harvested for total RNA using Tri Reagent according to the manufacturer's instructions. Concentrations of total RNA were measured by spectrophotometry 
(Nanodrop ND-100 Spectrophotometer, Biolab). Reverse Transcriptase-PCR was performed according to the manufacturer's protocol for SuperScript III First Strand cDNA Synthesis Kit (Invitrogen). Quantitative ('real-time') Reverse Transcriptase-PCR (QRT-PCR) was performed using SensiMix dT (Quantace) on a Corbett Rotorgene 3000 and analyzed using Rotor-Gene 6 Version 6.0 (Build 27) (Corbett Research). Primer pairs used for the amplification of HMG-CoA reductase from cDNAs are F- ttggtgatgggagcttgctgtg; R- agtcacaagcacgtggaagacg. The primer pair used for amplification of the housekeeping gene PBGD was described previously [25]. PCR products were verified by sequencing. The change in gene expression levels was determined by normalizing mRNA levels of the gene of interest to the mRNA level of the house-keeping gene, porphobilinogen deaminase (PBGD). Melting curve analysis was performed to confirm production of a single product in each reaction.

\section{Data Presentation and Statistics}

Data are presented as mean + standard error of the means (SEM) unless otherwise stated. All results are pooled or representative of at least two separate experiments. Statistical analyses were performed to find correlations (Pearson's) between two sets of continuous variables. A P-value less than 0.05 was considered statistically significant.

\section{Abbreviations}

24,25EC, 24(S),25-epoxycholesterol; ABCA1, ATP-binding cassette transporter-A1; HMG-CoA, 3-hydroxy-3methylglutaryl coenzyme A; LXR, liver $\times$ receptor; OSC, 2,3-oxidosqualene cyclase; SRE, sterol response element; SREBP, sterol regulatory element-binding protein; TLC, thin layer chromatography

\section{Additional material}

\section{Additional file 1}

Validation of TLC/phosphorimaging approach for determining cholesterol and 24,25EC synthesis. (C) CHO-7 cells were incubated with [1${ }^{14} \mathrm{Cl}$-acetate for $24 \mathrm{~h}$ under conditions of partial OSC inhibition to yield comparable levels of cholesterol and 24,25EC. Samples were pooled and neutral lipids extracted. Varying amounts of the extracts were applied to a thin-layer chromatography plate and the greatest amount was assigned an arbitrary relative concentration of 1 . After development, bands corresponding to authentic cholesterol and 24,25EC were visualized by phosphorimager and quantified by densitometry $(\boldsymbol{A}, \boldsymbol{B})$. Alternatively, bands were cut, eluted and quantified by scintillation counting $(D, E)$. Equations for the lines of best fit (A) $y=1.01 x ; R^{2}=0.99 ;(B) y=1.00 x ; R^{2}$ $=0.99 ;(D) y=1.00 x ; R^{2}=0.99 ;(E) y=1.00 x ; R^{2}=0.99$.

Click here for file

[http://www.biomedcentral.com/content/supplementary/1476511X-6-10-S1.pdf]

\section{Additional file 2}

24(S),25-epoxycholesterol synthesis is increased in HepG2 cells treated with GW534511X. HepG2 cells were incubated with increasing concentrations of GW534511X in the presence of [1-14 C]-acetate for 24 $h$. Neutral lipid extracts were separated by thin-layer chromatography and bands corresponding to authentic cholesterol and 24,25EC were visualized by phosphorimager.

Click here for file

[http://www.biomedcentral.com/content/supplementary/1476511X-6-10-S2.pdf]

\section{Acknowledgements}

We thank several researchers for sharing their valuable tools: Drs. Michael S. Brown, Joseph L. Goldstein, Malcolm Lyons, Alan Tall, and Glaxo SmithKline. We are grateful to Dr Ingrid Gelissen for critically reading this manuscript. This work was supported by a grant from the National Health and Medical Research Council (NHMRC\#350828). CMQ was supported by the NHMRC Atherosclerosis Program (222722).

\section{References}

I. Kandutsch AA, Chen HW, Heiniger HJ: Biological activity of some oxygenated sterols. Science 1978, 20I(4355):498-50I.

2. Goldstein JL, DeBose-Boyd RA, Brown MS: Protein sensors for membrane sterols. Cell 2006, I 24(I):35-46.

3. DeBose-Boyd RA, Ou J, Goldstein JL, Brown MS: Expression of sterol regulatory element-binding protein Ic (SREBP-Ic) mRNA in rat hepatoma cells requires endogenous LXR ligands. Proc Natl Acad Sci U S A 200I, 98(4): I477- I 482.

4. Janowski BA Shan, B., and Russell, D.W.: The Hypocholesterolemic Agent LY295427 Reverses Suppression of Sterol Regulatory Element-binding Protein Processing Mediated by Oxysterols. J Biol Chem 200I, 276:45408-454I6.

5. Tontonoz $P$, Mangelsdorf $D$ J: Liver $\mathbf{X}$ receptor signaling pathways in cardiovascular disease. Mol Endocrinol 2003, I 7(6):985-993.

6. Lehmann JM, Kliewer SA, Moore LB, Smith-Oliver TA, Oliver BB, Su JL, Sundseth SS, Winegar DA, Blanchard DE, Spencer TA, Willson TM: Activation of the nuclear receptor LXR by oxysterols defines a new hormone response pathway. J Biol Chem 1997, 272(6):3|37-3|40.

7. Spencer TA, Li D, Russel JS, Collins JL, Bledsoe RK, Consler TG, Moore LB, Galardi CM, McKee DD, Moore JT, Watson MA, Parks DJ, Lambert MH, Willson TM: Pharmacophore analysis of the nuclear oxysterol receptor LXRalpha. J Med Chem 200I, 44(6):886-897.

8. Schroepfer G] Jr.: Oxysterols: modulators of cholesterol metabolism and other processes. Physiol Rev 2000, 80(I):36I-554.

9. van Reyk DM, Brown AJ, Hult'en LM, Dean RT, Jessup W: Oxysterols in biological systems: sources, metabolism and pathophysiological relevance. Redox Rep 2006, I I (6):255-262.

10. Nelson JA, Steckbeck SR, Spencer TA: Biosynthesis of 24,25epoxycholesterol from squalene 2,3;22,23-dioxide. J Biol Chem 1981, 256(3): 1067-1068.

II. Saucier SE, Kandutsch AA, Taylor FR, Spencer TA, Phirwa S, Gayen AK: Identification of regulatory oxysterols, 24(S), 25-epoxycholesterol and 25-hydroxycholesterol, in cultured fibroblasts. J Biol Chem 1985, 260(27): | 1457|-14579.

12. Janowski BA, Grogan MJ, Jones SA, Wisely GB, Kliewer SA, Corey EJ, Mangelsdorf DJ: Structural requirements of ligands for the oxysterol liver $X$ receptors LXRalpha and LXRbeta. Proc Nat Acad Sci U S A 1999, 96(I):266-27I.

13. Wong J, Quinn CM, Brown AJ: Statins inhibit synthesis of an oxysterol ligand for the liver $x$ receptor in human macrophages with consequences for cholesterol flux. Arterioscler Thromb Vasc Biol 2004, 24( I 2):2365-237I.

14. Rowe AH, Argmann CA, Edwards JY, Sawyez CG, Morand $\mathrm{OH}$ Hegele RA, Huff MW: Enhanced synthesis of the oxysterol 
24(S),25-epoxycholesterol in macrophages by inhibitors of 2,3-oxidosqualene:lanosterol cyclase: a novel mechanism for the attenuation of foam cell formation. Circ Res 2003, 93(8):7| 7-725.

15. Dollis D, Schuber F: Effects of a 2,3-oxidosqualene-lanosterol cyclase inhibitor 2,3:22,23-dioxidosqualene and 24,25-epoxycholesterol on the regulation of cholesterol biosynthesis in human hepatoma cell line HepG2. Biochem Pharmacol 1994, 48(I):49-57.

16. Mark M, Muller P, Maier R, Eisele B: Effects of a novel 2,3-oxidosqualene cyclase inhibitor on the regulation of cholesterol biosynthesis in HepG2 cells. J Lipid Res 1996, 37(I): I48-I 58.

17. Venteclef N, Guillard R, Issandou M: The imidazoline-like drug S235 I 5 affects lipid metabolism in hepatocyte by inhibiting the oxidosqualene: lanosterol cyclase activity. Biochem Pharmacol 2005, 69(7): 104I-1048.

18. Eisele B, Budzinski R, Muller P, Maier R, Mark M: Effects of a novel 2,3-oxidosqualene cyclase inhibitor on cholesterol biosynthesis and lipid metabolism in vivo. J Lipid Res 1997, 38(3):564-575.

19. Zhang Z, Li D, Blanchard DE, Lear SR, Erickson SK, Spencer TA: Key regulatory oxysterols in liver: analysis as delta4-3-ketone derivatives by HPLC and response to physiological perturbations. J Lipid Res 200I, 42(4):649-658.

20. Morand OH, Aebi JD, Dehmlow H, Ji YH, Gains N, Lengsfeld H, Himber J: Ro 48-8.07I, a new 2,3-oxidosqualene:lanosterol cyclase inhibitor lowering plasma cholesterol in hamsters, squirrel monkeys, and minipigs: comparison to simvastatin. J Lipid Res 1997, 38(2):373-390

21. Telford DE, Lipson SM, Barrett PH, Sutherland BG, Edwards JY, Aebi JD, Dehmlow H, Morand OH, Huff MW: A novel inhibitor of oxidosqualene:lanosterol cyclase inhibits very low-density lipoprotein apolipoprotein BI00 (apoBI00) production and enhances low-density lipoprotein apoB 100 catabolism through marked reduction in hepatic cholesterol content. Arterioscler Thromb Vasc Biol 2005, 25(I 2):2608-26I4.

22. Bjorkhem I, Diczfalusy U: 24(S), 25-epoxycholesterol--a potential friend. Arterioscler Thromb Vasc Biol 2004, 24(I 2):2209-22I0.

23. Huff MW, Telford DE: Lord of the rings--the mechanism for oxidosqualene:lanosterol cyclase becomes crystal clear. Trends Pharmacol Sci 2005, 26(7):335-340.

24. Goldstein JL, Rawson RB, Brown MS: Mutant mammalian cells as tools to delineate the sterol regulatory element-binding protein pathway for feedback regulation of lipid synthesis. Arch Biochem Biophys 2002, 397(2): | 39-| 48.

25. Wong J, Quinn CM, Brown AJ: SREBP-2 positively regulates transcription of the cholesterol efflux gene, ABCAI by generating oxysterol ligands for LXR. Biochem / 2006, 400:485-49I.

26. Du X, Kristiana I, Wong J, Brown AJ: Involvement of Akt in ERto-Golgi transport of SCAP/SREBP: a link between a key cell proliferative pathway and membrane synthesis. Mol Biol Cell 2006, I I(6):2735-2745.

27. Briggs MR, Yokoyama C, Wang X, Brown MS, Goldstein JL: Nuclear protein that binds sterol regulatory element of low density lipoprotein receptor promoter. I. Identification of the protein and delineation of its target nucleotide sequence. J Biol Chem 1993, 268(19): I4490-14496.

28. Shimano $H$ : Sterol regulatory element-binding proteins (SREBPs): transcriptional regulators of lipid synthetic genes. Prog Lipid Res 200I, 40(6):439-452.

29. Venkateswaran A, Laffitte BA, Joseph SB, Mak PA, Wilpitz DC, Edwards PA, Tontonoz P: Control of cellular cholesterol efflux by the nuclear oxysterol receptor LXR alpha. Proc Natl Acad Sci U S A 2000, 97(22): 12097-12102.

30. Costet $P$, Luo $Y$, Wang N, Tall AR: Sterol-dependent transactivation of the $A B C I$ promoter by the liver $X$ receptor/retinoid X receptor. J Biol Chem 2000, 275(36):28240-28245.

31. Taylor FR, Kandutsch AA, Gayen AK, Nelson JA, Nelson SS, Phirwa $S$, Spencer TA: 24,25-Epoxysterol metabolism in cultured mammalian cells and repression of 3-hydroxy-3-methylglutaryl-CoA reductase. J Biol Chem 1986, 26I(32): I5039-15044.

32. Spencer TA, Gayen AK, Phirwa S, Nelson JA, Taylor FR, Kandutsch AA, Erickson SK: 24(S),25-Epoxycholesterol. Evidence consistent with a role in the regulation of hepatic cholesterogenesis. J Biol Chem 1985, 260(25): | 339|-13394.
33. Funk J, Landes C: Histopathologic findings after treatment with different oxidosqualene cyclase (OSC) inhibitors in hamsters and dogs. Exp Toxicol Pathol 2005, 57(I):29-38.

34. Goldstein JL, Basu SK, Brown MS: Receptor-mediated endocytosis of low-density lipoprotein in cultured cells. Methods Enzymol 1983, 98:24I-260.

35. Basu SK, Goldstein JL, Anderson GW, Brown MS: Degradation of cationized low density lipoprotein and regulation of cholesterol metabolism in homozygous familial hypercholesterolemia fibroblasts. Proc Natl Acad Sci U S A 1 976, 73(9):3। 78-3 I 82

36. Whitney KD, Watson MA, Goodwin B, Galardi CM, Maglich JM, Wilson JG, Willson TM, Collins JL, Kliewer SA: Liver X receptor (LXR) regulation of the LXRalpha gene in human macrophages. J Biol Chem 200I, 276(47):43509-435I5.

Publish with Bio Med Central and every scientist can read your work free of charge

"BioMed Central will be the most significant development for disseminating the results of biomedical research in our lifetime. "

Sir Paul Nurse, Cancer Research UK

Your research papers will be:

- available free of charge to the entire biomedical community

- peer reviewed and published immediately upon acceptance

- cited in PubMed and archived on PubMed Central

- yours - you keep the copyright
BioMedcentral 